\title{
Research on Multi-frequency Constant Envelope Multiplexing Technology of Satellite Navigation Signal
}

\author{
Li Rui \\ Department of Electronic Engineering \\ Tsinghua University \\ Beijing, China \\ liray@live.com \\ Zhang Peiyao \\ School of Information and Electronics \\ Beijing Institute of Technology \\ Beijing, China \\ zhangpy329@163.com
}

\author{
Zeng Dazhi \\ School of Information and Electronics \\ Beijing Institute of Technology \\ Beijing, China \\ zengdazhi@racobit.com \\ Lu Mingquan \\ Department of Electronic Engineering \\ Tsinghua University \\ Beijing, China \\ lumq@tsinghua.edu.cn
}

\begin{abstract}
Non-linear distortion would be introduced when non-constant envelope signal passes through power amplifiers. In the satellite navigation system, since composite signal generated by direct linear combination of signals does not have constant envelope characteristic, unexpected distortion would be introduced when the composite signal passes through the amplifier located in load transmitter. Therefore, constant envelope processing is necessary for multiple satellite signals of one certain frequency or different frequencies.In this paper, the existing constant envelope multiplexing techniques of multi-frequency, such as AltBOC, TD-AItBOC, POCET and ACED are studied and analyzed. The study shows that the AltBOC signal with constant envelope has strong anti-multipath capability and good tracking performance. On this basis, to realize the constant envelope multiplexing of $B 1$ and $L 1$ signals, a constant envelope modulation method based on AltBOC multiplexing technique is proposed. Simulation shows that this method can realize the constant envelope multiplexing of four signal components with two center frequencies.
\end{abstract}

Keywords: satellite navigation; multiplexing; constantenvelope; AltBOC

\section{INTRODUCTION}

The AM/AM and AM/PM distortion of the signal waveform would be introduced when the non-constant envelope signal passes through the non-linear power amplifier located in satellite load transmitter. Therefore, the transmitted signal should have constant-envelope characteristic. Signal with constant-envelope is not only good for transmission, but also good for the design and operation of the receiver. At present, the common multifrequency constant-envelope multiplexing technologies mainly include AltBOC, TD-AltBOC, ACED and POCET.

AltBOC modulation technology, proposed in 2000, is a kind of sub-carrier modulation method based on BOC modulation $^{[1]}$. However, as the four-channel AltBOC modulation signal does not have constant-envelope characteristic, it had not been applied. Due to the constant- envelope multiplexing implementation method of fourchannel AltBOC signal put forward by NCES in 2001, the modulation method was adopted by Galileo satellite navigation system in E5 frequency later. In order to solve the problem of limitation on channel power allocation of AltBOC technology, ACED technology was proposed. This kind of modulation method can allocate more power to the pilot channel. POCET technology was proposed by P.A.Dafesh in 2009, which can make multiple channel signals combined into a constant-envelope signal by carrier phase modulation ${ }^{[2][3][4]}$.

\section{MULTI-FREQUENCY CONSTANT ENVELOPE TECHNOLOGIES}

\section{A. AltBOC}

Compared to other signals, Alternate Binary Offset Carrier modulation signal has better anti-multipath capability and tracking performance ${ }^{[5]}$. It has been applied in Galileo E5 frequency band and China secondgeneration satellite navigation system B2 band. Usually, AltBOC $(n, m)$ is used to represent the AltBOC modulation signal, in which $n \times 10.23 \mathrm{MHz}$ represents carrier frequency and $m \times 10.23 \mathrm{MHz}$. represents the code rate. The expression of four-channel constant-envelope AltBOC signal is as follows.

$$
\begin{aligned}
s(t)= & \frac{1}{2 \sqrt{2}}\left[\sqrt { 2 P _ { 1 } } \left(\left(S_{A-d}(t)+j S_{A-p}(t)\right) S C_{8, S S B}{ }^{*}(t)+\right.\right. \\
& \left.\left(S_{B-d}(t)+j S_{B-p}(t)\right) S C_{8, S S B}(t)\right)+ \\
& \sqrt{2 P_{3}}\left(\left(S_{A-d}(t)+j \overline{S_{A-p}}(t)\right) S C 3_{8, S S B}{ }^{*}(t)+\right. \\
& \left.\left.\left.\overline{\left(S_{B-d}\right.}(t)+j S_{B-p}(t)\right) S C 3_{8, S S B}(t)\right)\right]
\end{aligned}
$$




$$
\begin{aligned}
& \overline{S_{A-d}}(t)=S_{A-p}(t) \cdot S_{B-d}(t) \cdot S_{B-p}(t) ; \\
& \overline{S_{A-p}}(t)=S_{A-d}(t) \cdot S_{B-d}(t) \cdot S_{B-p}(t) ; \\
& \overline{S_{B-d}}(t)=S_{A-d}(t) \cdot S_{A-p}(t) \cdot S_{B-p}(t) ; \\
& \overline{S_{B-p}}(t)=S_{A-d}(t) \cdot S_{A-p}(t) \cdot S_{B-d}(t) ;
\end{aligned}
$$

Where, $S_{A-d}(t), S_{A-p}(t), S_{B-d}(t)$ and $S_{B-p}(t)$ represent the data and pilot components of two frequencies respectively. $S C_{8, S S B}(t)$ and $S C 3_{8, S S B}(t)$ represent the subcarriers. A more detailed introduction of this method will be given in the next chapter.

\section{B. TD-AltBOC}

AltBOC modulation method can offer different services in the upper and the lower sidebands. However, this kind of modulation method has some defects such as the complexity in reception processing and low multiplexing efficiency. TD-AltBOC modulation technology can solve these problems to some extent and has similar spectral characteristics, interoperability and anti-multipath capability like AltBOC technology. The expressions of binary subcarrier adopted by TD-AltBOC are as follows.

$$
\begin{array}{ll}
S C_{B-d}(t)=\operatorname{sgn}\left[\cos \left(2 \pi f_{S C} t\right)\right]-j \operatorname{sgn}\left[\sin \left(2 \pi f_{S C} t\right)\right] & (2-6) \\
S C_{B-u}(t)=\operatorname{sgn}\left[\cos \left(2 \pi f_{S C} t\right)\right]+j \operatorname{sgn}\left[\sin \left(2 \pi f_{S C} t\right)\right] & (2-7)
\end{array}
$$

When TD-AltBOC modulation method is adopted, only two signals can be transmitted during the same time period and the type of the signal transmitted during odd and even slot has no effect on this method. During the same time period, there are three kinds of transmission scheme: (1) the transmission of two pilot components; (2) the transmission of two data components; (3) the transmission of one pilot component and one data component. The expression of baseband signal is as follows.

$$
\begin{aligned}
s(t)= & {\left[S_{A-d}(t)+S_{A-p}(t)\right] S C_{B-d}(t)+} \\
& {\left[S_{B-d}(t)+S_{B-p}(t)\right] S C_{B-u}(t) }
\end{aligned}
$$

Where, $S_{A-d}(t), S_{A-p}(t), S_{B-d}(t)$ and $S_{B-p}(t)$ represent the data and pilot components of two frequencies respectively.

\section{POCET}

POCET (Phase-Optimized Constant-Envelope Transmission) is a kind of technology which can make multichannel signals combined into a constant-envelope signal by carrier phase modulation. The basic principle of POCET technology is the phase modulation of the carrier. Considering the phase as a deterministic function of the binary signal chips, the phase vector is calculated by minimizing the envelope of the composite signal and stored in a lookup table. The modulated phase of the transmitted carrier is determined by the value of signal components to be transmitted according to the lookup table. When the number of signal components is changed, only the size of the lookup table need to be changed.

When POCET technology is adopted, the multiplexed signals should be uncorrelated and the probability of ' 0 ' and ' 1 ' level should be equal.
There are two restrictions when the optimal solution of the phase is calculated.

- Power restriction:

$$
P_{d n}=\left|\operatorname{corr}_{n}(\theta)\right|^{2}=\left|\frac{A}{2^{N}} \sum_{k=0}^{2^{N}-1}\left[1-2 b_{n}(k)\right] \exp \left(j \theta_{k}\right)\right|^{2}
$$

- Phase restriction:

$$
\begin{aligned}
& \operatorname{Im}\left\{e^{-j \Delta \phi_{n l}} \operatorname{corr}_{n}(\theta) \operatorname{corr}_{l}(\theta)^{*}\right\}=0 \\
& \operatorname{Re}\left\{e^{-j \Delta \phi_{n l}} \operatorname{corr}_{n}(\theta) \operatorname{corr}_{l}(\theta)^{*}\right\}>0
\end{aligned}
$$

Where, $\theta=<\theta_{0}, \ldots, \theta_{2^{N}-1}>$ is the vector composed of each phase value. $A$ is the constant-envelope value of the phase modulated carrier. $\theta_{k}\left(k \in 0 \sim 2^{N}-1\right)$ is the phase value of the signal in channel $k$ at time $t_{k} \cdot \operatorname{corr}_{n}(\theta)$ and $\operatorname{corr}_{l}(\theta)$ are the cross-correlation of spreading code and composite signal. $\Delta \phi_{n l}$ is the phase difference between signal in channel $n$ and channel $l$. Since the initial phase is arbitrary and the phase has symmetry characteristic, the phase can be calculated based on the following conditions.

$$
\theta_{0}=0 ;\left|\theta_{i}-\theta_{2^{N-i-1}}\right|=\pi \quad\left(0 \leq \theta_{i} \leq 2 \pi\right)
$$

\section{D. $A C E D$}

When the transmission power is fixed, ACED (Asymmetric Constant Envelope Double-sideband) technology can allocate different power to each sideband according to different demands. Therefore, more power can be allocated to pilot channel to improve the tracking performance.

Assuming that $c_{1}, c_{2}, c_{3}$ and $c_{4}$ represent the baseband spreading codes of the four signals, and these codes are orthogonal to each other. The signal of each channel can be expressed as $s_{i}(t)=A_{i} c_{i}(t) d_{i}(t) . A_{i}$ is the amplitude of channel $i$ and it can have different values in different channels. $d_{i}(t)$ is the data code modulated in channel $i$. The total power of all channels are normalized for convenience of calculation. The expression of the ACED signal is as follows.

$$
\begin{aligned}
s(t)= & \frac{\sqrt{2}}{2} \alpha_{1} \operatorname{sgn}\left[\sin \left(2 \pi f_{s c} t+\varphi_{1}\right)\right]+ \\
& j \frac{\sqrt{2}}{2} \alpha_{2} \operatorname{sgn}\left[\sin \left(2 \pi f_{s c} t+\varphi_{2}\right)\right] \\
\alpha_{1}= & -\sqrt{\left(s_{1}+s_{3}\right)^{2}+\left(s_{2}-s_{4}\right)^{2}} ; \\
\alpha_{2}= & \sqrt{\left(s_{1}-s_{3}\right)^{2}+\left(s_{2}+s_{4}\right)^{2}} ; \\
\varphi_{1}= & -a \tan 2\left[\left(s_{1}+s_{3}\right),\left(s_{2}-s_{4}\right)\right] ; \\
\varphi_{2}= & a \tan 2\left[\left(s_{2}+s_{4}\right),\left(s_{1}-s_{3}\right)\right] ;
\end{aligned}
$$

ACED has the advantages that the power does not need to be allocated equally so that more power can be allocated to pilot channel to improve ranging performance. Meanwhile, a certain channel can be closed by setting $\mathrm{A}_{i}$ as 0 without affecting the constant envelope characteristic. 
Thus, ACED technology can be adopted to realize constant envelope modulation of no more than four signals.

\section{COnStant EnVElope AltBOC Modulation SIMULATION ON B1AND L1 FREQUENCIES}

Considering the advantages of AltBOC technology, the constant-envelope simulation of B1 and L1 using AltBOC technology is realized in this paper. Next, the detail of AltBOC method and simulation results will be given.

The expression of four-channel AltBOC modulation baseband signal in the time domain is as follows.

$$
\begin{aligned}
s(t)= & \left(B_{A-d}(t)+j B_{A-p}(t)\right) \cdot S C_{4, S S B}{ }^{*}(t) \\
& +\left(B_{B-d}(t)+j B_{B-p}(t)\right) \cdot S C_{4, S S B}(t)
\end{aligned}
$$

Where, $B_{A-d}, B_{A-p}, B_{B-d}$ and $B_{B-p}$ represent the data and pilot components of two frequencies. The expression of compound subcarrier and the expressions of the two sub-carrier are as follows.

$$
\begin{aligned}
& S C_{4, S S B}(t)=\frac{1}{\sqrt{2}}(\operatorname{cr}(t)+j \cdot \operatorname{sr}(t)) \\
& \operatorname{cr}(t)=\operatorname{sign}\left(\cos \left(2 \pi f_{s c} t\right)\right) \\
& \operatorname{sr}(t)=\operatorname{sign}\left(\sin \left(2 \pi f_{s c} t\right)\right)
\end{aligned}
$$

According to the expression of four-channel AltBOC modulation baseband signal, when the number of ' 1 ' and ' -1 ' are even among four ranging codes, the phase only varies between phase 1 and 5 or phase 3 and 7 . When the number of ' 1 ' and '- 1 ' are odd among four ranging codes, the phase only varies between phase 4,0 and 8 or phase 2,0 and 6 . The signal has different amplitudes in odd phase and even phase and has 0 -phase, which means the signal does not have constant envelope. Thus, the constantenvelope processing is needed. Taking B1 with the center frequency of $1561.098 \mathrm{MHz}$ and $\mathrm{L} 1$ with the center frequency of $1575.42 \mathrm{MHz}$ as an example, the signal modulated by $\operatorname{AltBOC}(7,2)$ is shown in Fig .1. Fig .1(a) shows the amplitude of baseband signal and Fig .1(b) shows the waveform after QPSK modulation on the carrier. It is obvious that the signal does not have constant envelope.

In order to eliminate the 0 -phase, the signal amplitude is extended to both sides by $1 / 8$ cycle when the amplitude is $\pm 2 \sqrt{2}$. In order to keep the same power, the amplitude is set to $1 / \sqrt{2}$ of the original. After the processing, the 0 phase is eliminated and the amplitude is the same for odd phase and even phase. So the signal has the constantenvelope characteristic. To achieve the process above, the vector decomposition of the subcarriers is needed.

After decomposition, the expressions of sub-carriers are as follows.

$$
\begin{aligned}
& S C_{8, S S B}(t)=\frac{1}{\sqrt{2 P_{1}}}\left[s c_{E 5-s}(t)+j s c_{E 5-s}\left(t-T_{s, E 5} / 4\right)\right] \\
& S C 3_{8, S S B}(t)=\frac{1}{\sqrt{2 P_{3}}}\left[s c_{E 5-p}(t)+j s c_{E 5-p}\left(t-T_{s, E 5} / 4\right)\right]
\end{aligned}
$$
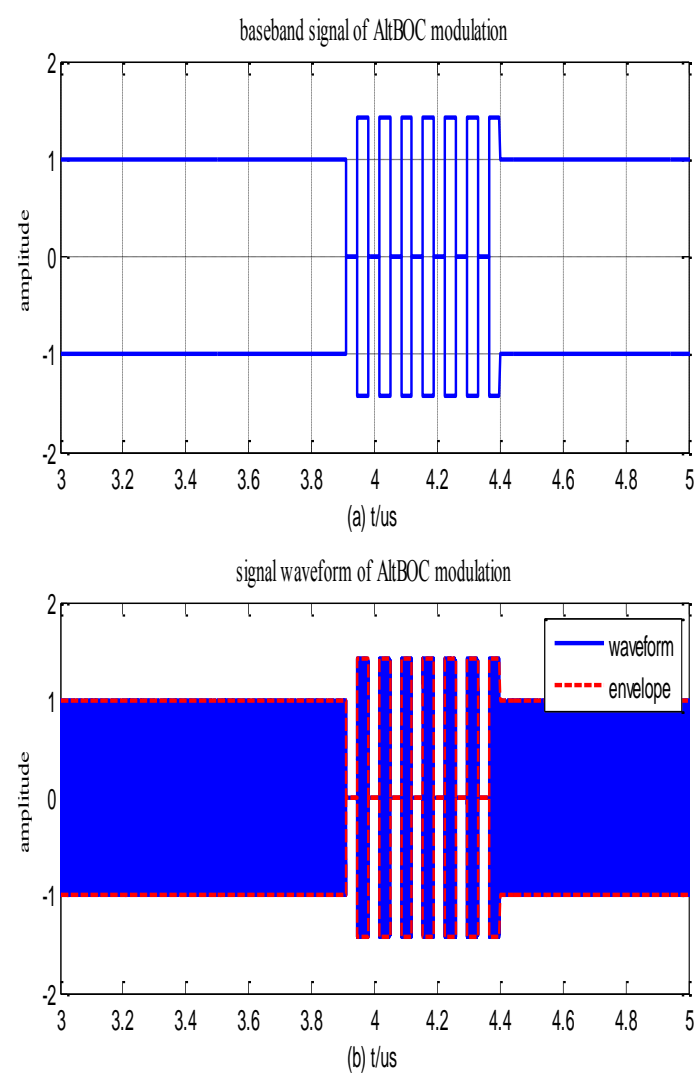

Figure 1. AltBOC $(7,2)$ modulation for $\mathrm{B} 1$ and $\mathrm{L} 1$ signals

The values of sub-carrier $s c_{E 5-s}(t)$ are obtained from eight sample values in a cycle of cosine signal multiplied by a factor. The eight values of $s c_{E 5-s}(t)$ and $s c_{E 5-p}(t)$ in a cycle are shown in Table 1.

According to the analysis above, the ultimate expression of AltBOC signal shown in equ.(2-1) is proved.

As the AltBOC signal does not only has the advantages of BOC signals, such as spectral separation, antiinterference ability, high ranging precision and so on, but also can compensate for the defection of BOC signal that the two main lobes can only transmit the same information. Meanwhile, the intermodulation terms introduced do not affect the useful signal power. The receiver can receive and process the single sideband signal independently or the two sidebands signal together to improve the positioning accuracy. Considering all these advantages, the AltBOC constant-envelope modulation simulation on B1 with center frequency of $1561.098 \mathrm{MHz}$ and L1 with center frequency of $1575.42 \mathrm{MHz}$ is realized in this paper.

According to the principle of AltBOC modulation, it is known that in order to modulate the $\mathrm{B} 1$ signal and L1 signal on the same carrier using AltBOC technology, the frequency of the carrier should be $1568.529 \mathrm{MHz}$ and the frequency of complex sub-carrier should be $7.161 \mathrm{MHz}$. Therefore, AltBOC $(7,2)$ is adopted to realize the constant envelope modulation. The simulation results are shown in Fig .2. Fig . 2(a) shows the amplitude of the baseband signal. Fig .2(b) shows the waveform after modulating on the carrier. Fig .2(c) shows the waveform during 0.01us to show the detail more clearly. 


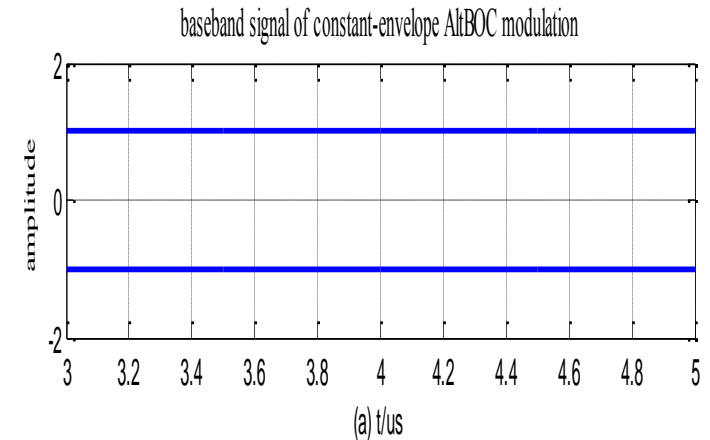

signal waveform of constant-enrebope AtBDCC modulation

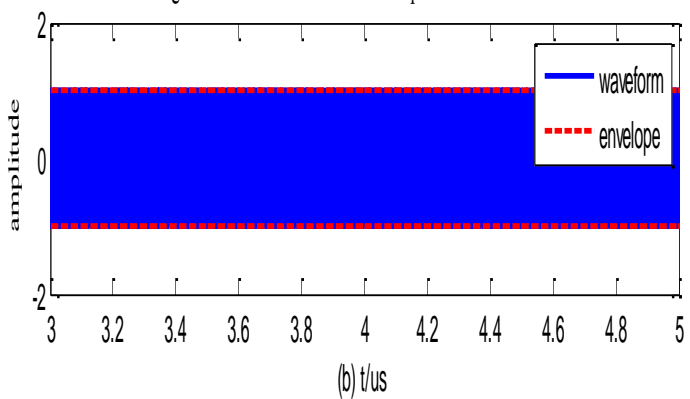

signal waveform of constant-enrelope ALBOC modulation

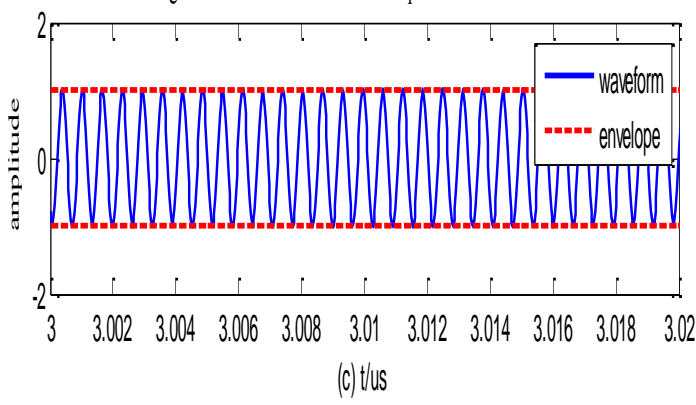

Figure 2. Constant-envelope AltBOC $(7,2)$ modulation for B1 and L1 signals

According to the figures, it is obvious that the signal has constant envelope.

Table I

\begin{tabular}{|c|c|c|c|c|}
\hline$i$ & 0 & 1 & 2 & 3 \\
\hline $2 A S_{i}$ & $\sqrt{2}+1$ & 1 & -1 & $-\sqrt{2}-1$ \\
\hline $2 A P_{i}$ & $-\sqrt{2}+1$ & 1 & -1 & $\sqrt{2}-1$ \\
\hline$i$ & 4 & 5 & 6 & 7 \\
\hline
\end{tabular}

\begin{tabular}{|l|l|l|l|l|}
\hline $2 A S_{i}$ & $-\sqrt{2}-1$ & -1 & 1 & $\sqrt{2}+1$ \\
\hline $2 A P_{i}$ & $\sqrt{2}-1$ & -1 & 1 & $-\sqrt{2}+1$ \\
\hline
\end{tabular}

\section{CONCLUSIONS}

To realize the constant envelope modulation of $\mathrm{B} 1$ and L1 signals, a kind of method using AltBOC modulation technology is proposed in this paper. Its main advantage is that the modulation and demodulation methods are relatively simple compared with other complex signal modulation methods. Moreover, the AltBOC signal has a narrow correlation peak which can improve the pseudorange tracking accuracy. Besides, the intermodulation items introduced to realize constant envelope has no effect on the power of desired signal. Simulation shows that $\operatorname{AltBOC}(7,2)$ method can realize the constant envelope modulation of B1 and L1 signals. It is hoped that the research in this paper can offer some help for solving the problem of multi-frequency constant envelope multiplexing.

\section{REFERENCES}

[1] Lei Zhiyuan,Guo Ji,LU Xiaochun, “Analysis of constant envelope alternate binary offset carrier modulation[J]" Journal of Time and Frequency,vol. 36, Jan. 2013,pp.44-53.

[2] Liu Yan, Li Chuanzhang,Niu Huifeng, "Research and Implementation of POCET Constant Envelope Modulation Technique[J]" Radio Communications Technology,vol. 39,2013,pp.85-88.

[3] Dafesh.P.A, Chan.C.R. Phase-Optimized Costant-Envelope Transmission(POCET) Modulation Method for GNSS Signals[C]. Proceedings of the $22^{\text {nd }}$ International Technical Meeting of the Satellite Division of the Institute of Navigation Savannah, Geogia,2009:2860-2866.

[4] Dafesh.P.A, Chan.C.R. Application of POCET Method to Combine GNSS Signals at Different Carreier Frequencies[C]. Proceedings of the 24nd International Technical Meeting of the Satellite Division of the Institute of Navigation,San Diego,California,2011:12011206 .

[5] Wang Haifeng, Huang Zhigang,Wang Wei, Xu Bingqi, Geng Shengqun. "Simulation and Performance Evaluation of AltBOC Signal[C]". The $2^{\text {nd }}$ China Satellite Navigation Conference. 2011.

[6] Xie Gang. Principles of GNSS:GPS,GLONASS, and Galileo[M] Beijing: Publishing House of Electronics Industry, 2013,pp:53-54

[7] Tang Zuping, Zhou Hongwei, Wei Jiaolong el al. "TD-AltBOC:A new COMPASS B2 Modulation[J]",Science China, vol.54,June 2011,pp:1015-1021.

[8] Zhu Liang, Yao Zheng, Lu Mingquan, Feng Zhenming. "Nonsymmetrical AltBOC multiplexing for Compass B1 signal design[J]". J Tsinghua Univ, 2012,vol:52,pp:869-873.

[9] Wang Lu, Liu Chonghua, Han Zhigang, Dong Haiqing. Study of Constant-envelope sinal combining methodologies for navigation system[C]. The $1^{\text {st }}$ China Satellite Navigation Conference.2010.

[10] Li Xianqiu, Luo Xianzhi, Li Jun. A New Way to Deduce Power Spectrum of ALTBOC Modulation Signal[J]. Ratio Engineering, 2012,vol.5,pp:34-35,64. 